\title{
Shikonin Inhibits Der p 2-Induced Cytokine and Chemokine Expression in Dendritic Cells in Patients with Atopic Dermatitis
}

\author{
Chung-Yang Yen, ${ }^{1}$ Wen-Dee Chiang, ${ }^{2}$ Shang-Yong Liu, ${ }^{2}$ Sheng-Jie Yu ${ }^{\mathbb{D}},{ }^{1,3}$ \\ and Ching-Liang Hsieh (iD) ${ }^{4,5,6}$ \\ ${ }^{1}$ Department of Dermatology, Taichung Veterans General Hospital, Taichung 40705, Taiwan \\ ${ }^{2}$ Department of Food Science, Tunghai University, Taichung 40704, Taiwan \\ ${ }^{3}$ Department of Education and Research, Kaohsiung Veterans General Hospital, Kaohsiung 81300, Taiwan \\ ${ }^{4}$ Chinese Medicine Research Center, China Medical University, Taichung 40402, Taiwan \\ ${ }^{5}$ Graduate Institute of Acupuncture Science, College of Chinese Medicine, China Medical University, Taichung 40402, Taiwan \\ ${ }^{6}$ Department of Chinese Medicine, China Medical University Hospital, Taichung 40447, Taiwan
}

Correspondence should be addressed to Sheng-Jie Yu; jim0929@msn.com and Ching-Liang Hsieh; clhsieh@mail.cmuh.org.tw

Received 20 March 2020; Revised 7 July 2020; Accepted 18 July 2020; Published 2 August 2020

Academic Editor: Isabel Andújar

Copyright (C) 2020 Chung-Yang Yen et al. This is an open access article distributed under the Creative Commons Attribution License, which permits unrestricted use, distribution, and reproduction in any medium, provided the original work is properly cited.

\begin{abstract}
Atopic dermatitis (AD) is a common inflammatory skin disorder. Shikonin, the active component of Lithospermum erythrorhizon extract, exhibits anti-inflammatory effects. The objective of the present study was to investigate the effect of shikonin on proinflammatory cytokines and chemokine in patients with AD. Ten patients with AD who were allergic to house dust mite (HDM) and seven healthy controls were recruited in this study. Peripheral blood mononuclear cells were isolated, and CD $14^{+}$cells were further selected and differentiated to dendritic cells. Dendritic cells stimulated using Der p 2, the major HDM allergen, were cotreated with shikonin for 24 hours, and dexamethasone was used as a control. Culture supernatants were collected, and proinflammatory cytokine and chemokine concentrations were analyzed using a multiplex assay system. Shikonin significantly inhibited Der p 2-induced expression of interleukin (IL)-6, IL-9, and IL-17A; monocyte chemoattractant protein (MCP)-1; macrophage inflammatory protein (MIP)- $1 \alpha$; MIP- $1 \beta$; and Chemokine (C-C motif) ligand 5 (RANTES). The inhibitory effects of shikonin on IL-9, MIP-1 $\beta$, and RANTES expression were stronger than those of dexamethasone. Therefore, Shikonin can be considered a promising drug for $\mathrm{AD}$ treatment because it inhibits different inflammatory cytokines expression.
\end{abstract}

\section{Introduction}

Atopic dermatitis (AD) is usually a lifelong disorder and requires long-term treatment. $\mathrm{AD}$ is a chronic, complex, and often relapsing inflammatory skin disorder. Pruritus, xerosis, and eczematous lesions are often observed in patients with $\mathrm{AD}[1]$. The prevalence of $\mathrm{AD}$ has been reported to vary from $0.3 \%$ to $6.2 \%$ in Europe, $3.2 \%$ to $10.7 \%$ in the United States, and $2.9 \%$ in Japan [2-4]. Itching and pain disrupt daily activities and sleep in patients with AD, which adversely affect their quality of life. Furthermore, mood changes, social isolation, and depression were reported to affect patients with moderate to severe $\mathrm{AD}$ [5]. High risks of comorbidities, such as malignancies, autoimmune diseases, cardiovascular diseases, and neuropsychiatric diseases, and an increase in economic burden, are correlated with the severity of $\mathrm{AD}$ [6]. In the United States, the estimated cost of treating $\mathrm{AD}$ was reported to be more than five billion dollars per year [7]. Hence, developing effective new drugs is a promising method to reduce the cost of treating $\mathrm{AD}$.

Thus far, medications used for treating AD were mainly used to control itching, repair skin, and ameliorate skin inflammation and infection. Topical application of calcineurin inhibitors, steroids, and antibiotics, as well as light therapy, was used as first-line medication. Oral steroids and immunosuppressive drugs effectively reduced the symptoms 
of moderate to severe AD [8]. Recently, the use of biological agents against interleukin (IL)- $4 \mathrm{R} \alpha$ was reported to treat $\mathrm{AD}$ [9]. However, the long-term use of steroids and immunosuppressive drugs exhibited side effects, including thinning of the skin, increased systemic infection, osteoporosis, and potential cancer risk. Moreover, high costs caused poor treatment persistence, and poor treatment adherence contributed to the adverse effects of inadequate treatment [10]. Therefore, the development of alternative low-cost remedies with minimal side effects, which are suitable for long-term use, is necessary.

In the skin of patients with $\mathrm{AD}$, the infiltration of immune cells, including dendritic cells, macrophages, Th2 cells, Th17 cells, and eosinophils, was observed. Crosstalk among these immune cells is reported to constitute the pathogenesis of AD. Among the immune cells, dendritic cells and macrophages were reported to play the most crucial role in initiating a Th2-cell immune response and skin inflammation [11]. FceRI expressed on the surface of dendritic cells was reported to bind with IgE; after challenge with the allergen, the number of dendritic cells increases in the epidermis and dermis, and the dendritic cells produce proinflammatory cytokines to enhance inflammation and differentiation of Th2 cells [12]. In AD, macrophages accumulate in the skin tissue and serve as antigen-presenting phagocytes against pathogens on the skin. During skin inflammation, macrophages produce proinflammatory cytokines and growth factors to enhance inflammatory responses [13]. Thus, inhibiting the activation of dendritic cells and macrophages has become the primary target for developing new drugs for treating $\mathrm{AD}$.

In our previous report, we demonstrated that Tzu-Yun ointment (TYO) effectively reduced scores on the Eczema Area and Severity Index (EASI) and Three Item Severity in patients with $\mathrm{AD}$ after 8 weeks of treatment [14]. The major active component of TYO is shikonin, which is isolated from the root of a traditional Chinese herb, Lithospermum erythrorhizon. The topical application of TYO for 8 weeks effectively ameliorated dermatitis in patients with $\mathrm{AD}$ (Figure 1). Shikonin was reported to inhibit ovalbumininduced activation of dendritic cells and attenuate allergic airway hyperresponsiveness in a murine model [15]. Furthermore, shikonin has been reported to reduce inflammation through antioxidation and inhibit chemotaxis in mononuclear macrophages and expression of genes related to lipopolysaccharide (LPS)-induced inflammation in macrophage cell lines [16-18]. These results suggest that shikonin exhibits multiple inhibitory effects on immune cells. However, information regarding the effects of shikonin on dendritic cells in patients with $\mathrm{AD}$ is not currently available.

In this study, we investigated the potential therapeutic effects and detailed mechanism of action of shikonin through its regulatory effects on the expression of proinflammatory cytokines and chemokines in dendritic cells. Understanding the therapeutic effects of shikonin on $A D$ treatment can provide hope for new drug development with fewer side effects and a lower cost than those of currently available drugs.

\section{Materials and Methods}

2.1. Participants. A total of 10 patients with $\mathrm{AD}$ and 7 healthy controls were enrolled in this study. Patients with $\mathrm{AD}$ were included if they met the criteria of Hanifin and Rajka [19] including pruritus, typical morphology and distribution, chronic or chronically relapsing dermatitis, and personal or family history of atopy. All patients with $\mathrm{AD}$ were sensitive to HDM, and HDM-specific IgE was measured by using the Bio IC system (Agnitio, Taiwan). All participants were selected from the clinic of the Dermatology Department of Taichung Veterans General Hospital.

2.2. Ethics Statement. All participants provided written informed consent. The protocols and all research involving human participants were approved by the Institutional Review Board of Taichung Veterans General Hospital (TCVGH-CF-13038A).

2.3. Chemicals. Purified recombinant protein Der $\mathrm{p} 2$ was purchased from Indoor Biotechnologies (Charlottesville, Virginia, USA). Shikonin was purchased from SigmaAldrich (St. Louis, MO, USA).

2.4. Cell Culture. For peripheral blood mononuclear cell (PBMC) culture, $16 \mathrm{~mL}$ of blood was collected from the patients in sodium citrate tubes (Vacutainer ${ }^{\circledR} \mathrm{CPT}^{\mathrm{TM}}, \mathrm{BD}$, USA), and PBMCs were purified through centrifugation over a density gradient. CD $14^{+}$cells were negatively selected using an MACS microbead column according to the manufacturer's protocol (Miltenyi Biotec, USA). Cells were cultured in RPMI-1640 supplemented with $10 \%$ fetal bovine serum, $1 \%$ penicillin/streptomycin, $25 \mathrm{mM}$ HEPES, and $2 \mathrm{mM} \mathrm{L}$-glutamine. CD14 ${ }^{+}$cells were further differentiated to obtain dendritic cells by stimulation with $50 \mathrm{ng} / \mathrm{mL}$ of recombinant granulocyte macrophage colony-stimulating factor (GM-CSF; PeproTech, USA) and $10 \mathrm{ng} / \mathrm{mL}$ of recombinant human IL-4 (PeproTech, USA) in a 6-well plate at $37^{\circ} \mathrm{C}$ and $5 \% \mathrm{CO}_{2}$ for 7 days. Nonadherent dendritic cells were removed using phosphate-buffered saline, and the remaining dendritic cells were collected for subsequent experiments. A total of $6 \times 10^{5}$ cells $/ \mathrm{mL}$ were cultured in a 12 -well plate and treated for 24 hours with the following: $1.5 \mu \mathrm{g} / \mathrm{mL}$ of Der p 2, $1.5 \mu \mathrm{g} / \mathrm{mL}$ of Der p 2, and $50 \mathrm{ng} / \mathrm{mL}$ shikonin, or $1.5 \mu \mathrm{g} / \mathrm{mL}$ of Der p 2 and $10^{-7} \mathrm{M}$ dexamethasone. The supernatant was collected for the subsequent measurement.

Cell viability was determined using the trypan blue dye exclusion assay.

Dendritic cells were treated with $0.016,0.05$, and $0.15 \mu \mathrm{g} /$ $\mathrm{mL}$ of shikonin for 24 hours. After treatment, cells were centrifuged and suspended in culture medium and mixed with trypan blue (Biological Industries, Kibbutz Beit Haemek, Israel) at a ratio of $1: 1$. A hemocytometer was used to count cells. Cell viability was calculated as the number of viable cells divided by the total number of cells [20]. 


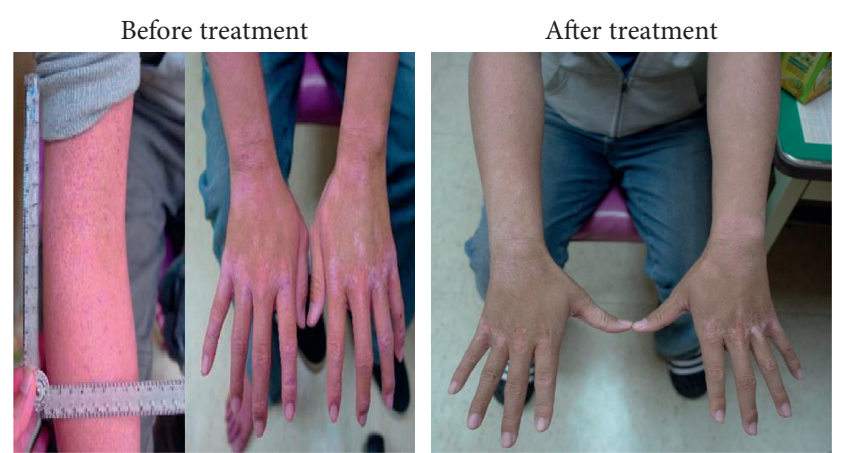

(a)

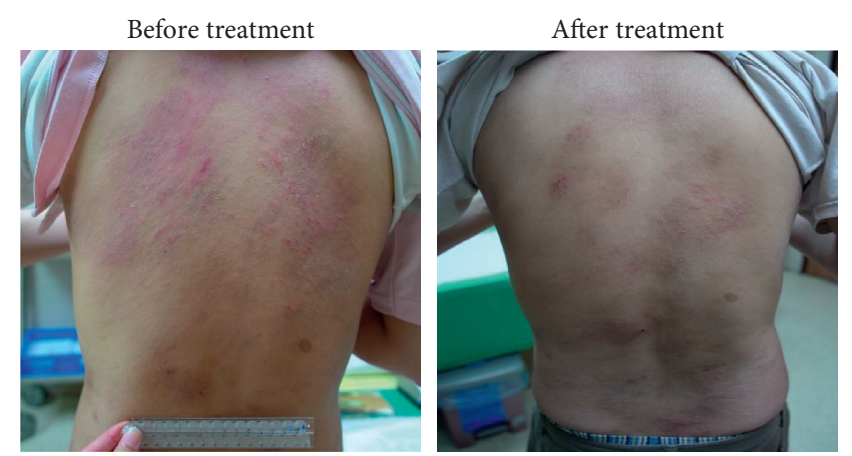

(b)

Figure 1: Effect of Tzu-Yun ointment (TYO) on patients with AD. (a) A 23-year-old man and (b) A 26-year-old man (left panel, before treatment) showed marked improvement in erythema and swelling after 8 weeks of treatment with topical application of TYO (right panel, after treatment).

2.5. Multiplex Assay. For measuring cytokine and chemokine levels, culture supernatants were collected and the concentrations of IL-1 $\beta$, IL-1ra, IL-2, IL-4, IL-5, IL-6, IL-7, IL-8, IL-9, IL-10, IL-12, IL-13, IL-15, and IL-17A; eotaxin; basic fibroblast growth factor; granulocyte colony-stimulating factor; GM-CSF; interferon- $\gamma$; interferon $\gamma$-induced protein-10; monocyte chemoattractant protein (MCP)1 (MCAF); macrophage inflammatory protein (MIP)- $1 \alpha$ and MIP-1 $\beta$; platelet-derived growth factor-BB; Chemokine (C$\mathrm{C}$ motif) ligand 5 (RANTES); tumor necrosis factor (TNF)$\alpha$; and vascular endothelial growth factor were determined using a protein multiplex immunoassay system (Bio-Plex Cytokine Array System, Bio-Rad Laboratories, Hercules, CA, USA).

2.6. Statistical Analyses. All statistical analyses were performed using SPSS, version 22 (IBM, Chicago, IL) by the Biostatistics Task Force of Taichung Veterans General Hospital, Taichung, Taiwan. The Wilcoxon signed-rank test was used, and data are presented as the mean \pm standard deviation (SD); $p<0.05$ was considered statistically significant.

\section{Results}

3.1. Comparison of Cytokine Profiles between Patients with AD and Healthy Controls. In this study, 10 patients with $\mathrm{AD}$ and 7 healthy controls were enrolled. Six of the 10 patients received melone, and all the 10 patients received topical steroid and oral histamine for control AD. Demographic characteristics, namely sex, age, asthma, allergic rhinitis status, EASI score, total IgE concentration, mite-specific $\operatorname{IgE}$ concentration, steroid usage, and TYO usage, were recorded (Tables 1 and 2). Dendritic cells were isolated and cultured for 24 hours. Subsequently, supernatants were collected for the cytokine profile analysis. The focus was mainly on innate cytokine and chemokine expression and cytokine and chemokine expression related to Th2 cells, Th17 cells, and macrophages.

Without Der p 2 induction, IL-1ra was significantly higher in the AD patients than in the controls $(p<0.05)$.
TABle 1: Demographic and clinical characteristics.

\begin{tabular}{lcc}
\hline & $\begin{array}{c}\text { Healthy subject } \\
(n=7)\end{array}$ & $\begin{array}{c}\text { AD patients } \\
(n=10)\end{array}$ \\
\hline Sex & $5(71.4)$ & $6(60 \%)$ \\
$\quad$ Male & $2(28.6)$ & $4(40 \%)$ \\
Female $(y r s)$ & $41.1 \pm 17.5$ & $29.8 \pm 11.1$ \\
EASI score & N. A & $15.3 \pm 11.7$ \\
IgE (IU/mL) & N. A & $3335.2 \pm 1779.2$ \\
Mite-specific IgE & N. A & $10(100 \%)$ \\
Topical steroid & $0(0 \%)$ & $10(100 \%)$ \\
treatment & & \\
Systemic steroid & $0(0 \%)$ & $6(60 \%)$ \\
treatment & $0(0 \%)$ & $5(50 \%)$ \\
Asthma & $2(28.6)$ & $6(60 \%)$ \\
Rhinitis & $0(0 \%)$ & $0(0 \%)$ \\
HBsAg & $0(0 \%)$ & $1(10 \%)$ \\
Anti-HCV & $0(0 \%)$ & $0(0 \%)$ \\
Autoimmune disease & $1(14.2 \%)$ & $0(0 \%)$ \\
Diabetes mellitus & $1(14.2 \%)$ & $0(0 \%)$ \\
Hypertension &
\end{tabular}

Values are expressed as the number (percentage) or mean \pm standard deviation.

Th2-dominant cytokine expression (IL-4, IL-9, and IL-13) levels were higher in the AD patients than in the controls $(p>0.05)$. Macrophage-related cytokine expression (MIP$1 \alpha$ and MIP- $1 \beta$ ) levels were higher in the AD patients than in the controls $(p>0.05)$. IL-7 is produced by keratinocytes, dendritic cells, neurons, and other epithelial cells. The IL-7 level was higher in the $\mathrm{AD}$ patients than in the controls $(p>0.05)$. The levels of IL-17A and RANTES were higher in the controls than in the $\mathrm{AD}$ patients $(p>0.05)$. The aforementioned effect might have been observed because some patients received oral prednisolone (Table 3).

3.2. Proinflammatory Cytokine Expression Reduced in the Patients after Treatment with Shikonin. In total, 27 cytokines were analyzed from the supernatants of dendritic cell cultures, and we found that Der p 2 significantly induced the expression of IL-1 $\beta$, IL-1ra, IL-6, IL-8, IL-9, IL-15, and IL- 


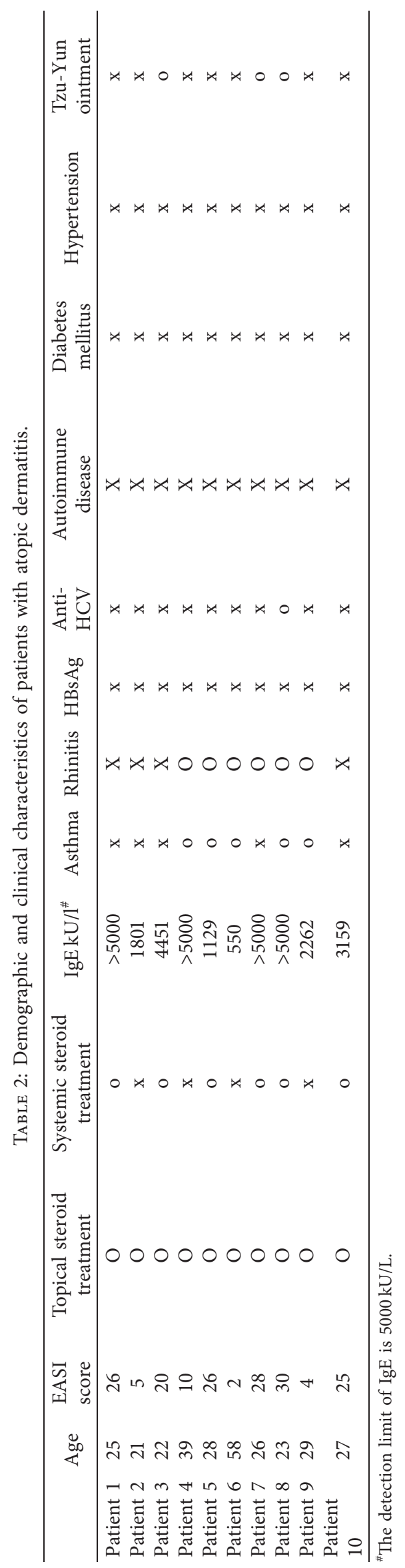


TABLe 3: Basal level of the cytokine profile from healthy controls and patients with atopic dermatitis.

\begin{tabular}{|c|c|c|c|c|c|}
\hline & \multicolumn{2}{|c|}{$\begin{array}{l}\text { Healthy subject } \\
\quad(n=7)\end{array}$} & \multicolumn{2}{|c|}{$\begin{array}{c}\text { AD patient } \\
(n=10)\end{array}$} & \multirow[t]{2}{*}{$p$ value } \\
\hline & Mean & SD & Mean & SD & \\
\hline IL- $1 \beta$ & 0.4 & \pm 0.4 & 1.1 & \pm 1.6 & 0.732 \\
\hline IL-1ra & 312.3 & \pm 571.7 & 680.5 & \pm 612.1 & $0.040^{*}$ \\
\hline IL-2 & 1.9 & \pm 1.5 & 2.2 & \pm 1.3 & 0.557 \\
\hline IL-4 & 0.3 & \pm 0.2 & 0.5 & \pm 0.4 & 0.435 \\
\hline IL-5 & 0.8 & \pm 1.2 & 0.8 & \pm 1.2 & 0.913 \\
\hline IL-6 & 1.5 & \pm 1.2 & 1.2 & \pm 0.7 & 0.733 \\
\hline IL-7 & 0.7 & \pm 1.9 & 3.2 & \pm 4.8 & 0.101 \\
\hline IL-8 & 20.7 & \pm 5.2 & 70.2 & \pm 87.4 & 0.845 \\
\hline IL-9 & 7.0 & \pm 5.5 & 12.3 & \pm 12.2 & 0.526 \\
\hline IL-10 & 2.0 & \pm 1.0 & 2.7 & \pm 1.7 & 0.435 \\
\hline IL-12 ( $p 70)$ & 3.0 & \pm 2.1 & 2.1 & \pm 1.8 & 0.464 \\
\hline IL-13 & 0.3 & \pm 0.4 & 1.5 & \pm 2.1 & 0.157 \\
\hline IL-15 & 9.6 & \pm 8.9 & 10.9 & \pm 9.2 & 0.696 \\
\hline IL-17A & 11.5 & \pm 5.4 & 7.3 & \pm 3.8 & 0.051 \\
\hline Eotaxin & 2.7 & \pm 1.6 & 3.0 & \pm 2.3 & 0.884 \\
\hline Basic FGF & 27.7 & \pm 11.4 & 30.8 & \pm 19.6 & 0.626 \\
\hline G-CSF & 11.4 & \pm 19.8 & 12.4 & \pm 9.3 & 0.187 \\
\hline GM-CSF & 92.3 & \pm 44.4 & 90.7 & \pm 65.0 & 0.626 \\
\hline IFN- $\gamma$ & 4.3 & \pm 6.4 & 5.9 & \pm 3.7 & 0.239 \\
\hline IP-10 & 1.4 & \pm 2.7 & 1.2 & \pm 2.0 & 0.819 \\
\hline MCP-1 & 122.7 & \pm 76.4 & 66.8 & \pm 63.8 & 0.079 \\
\hline MIP- $1 \alpha$ & 15.0 & \pm 17.1 & 25.2 & \pm 29.7 & 0.558 \\
\hline MIP-1 $\beta$ & 45.9 & \pm 44.2 & 81.8 & \pm 98.8 & 0.922 \\
\hline PDGF-BB & 3.7 & \pm 2.1 & 5.9 & \pm 5.9 & 0.695 \\
\hline RANTES & 17.3 & \pm 35.6 & 5.5 & \pm 2.5 & 0.406 \\
\hline TNF- $\alpha$ & 6.3 & \pm 10.6 & 2.6 & \pm 1.8 & 0.806 \\
\hline VEGF & 28.0 & \pm 35.6 & 19.7 & \pm 23.3 & 0.625 \\
\hline
\end{tabular}

Data are expressed as the mean \pm standard deviation. Data were analyzed using the Wilcoxon signed-rank test.

17; eotaxin; MCP-1; MIP- $1 \alpha$; MIP- $1 \beta$; RANTES; and TNF- $\alpha$ compared with basal levels (data not shown). Before determining the effects of shikonin on proinflammatory cytokine production, the toxicity of shikonin on dendritic cells was measured using the trypan blue exclusion assay. Results showed that no toxic effects were detected after dendritic cells were cocultured with $0.016,0.05$, and $0.15 \mu \mathrm{g} / \mathrm{mL}$ of shikonin for 24 hours (Figure 2).

Results also showed that in the $\mathrm{AD}$ patients, the levels of IL-1ra, IL-15, IL-17A, and MCP-1 were significantly lower in the shikonin-treated group than in the Der $\mathrm{p} 2$-stimulated group $(n=10)$. The levels of IL- 8, MIP- $1 \alpha$, MIP- $1 \beta$, and RANTES were also lower in the shikonin-treated group than in the Der $\mathrm{p} 2$-stimulated group; however, the difference was not significant. The expression levels of all cytokines were significantly lower in the dexamethasone-treated group than in the Der p 2-stimulated group (Figure 3). According to these results, 7 of the 10 patients were sensitive to shikonin treatment (patient number 4-10). By contrast, dendritic cells isolated from the remaining three patients exhibited higher levels of inflammatory cytokines after shikonin treatment than before treatment (patient number 1-3). We confirmed that no differences were observed in sex; EASI score; drug history; and combined other atopic disease, such as asthma or rhinitis, between the shikonin-sensitive patients and the other three patients (data not shown). Based on the effect of shikonin, three groups were distinguished (worse than prednisolone N1-3, better than or equal to prednisolone N4, and excellent response over prednisolone N5-10). We further analyzed the data in the excellent-response group, and results showed that Th2, Th17, and macrophage-dominant cytokines (namely, IL-6, IL-9, IL-17, MCP-1, MIP-1 $\alpha$, MIP$1 \beta$, and RANTES) were significantly downregulated after shikonin treatment (Table 4). Dexamethasone mainly inhibited Der p 2-induced proinflammatory cytokine expression (Table 5). Moreover, we compared the levels of proinflammatory cytokines between shikonin- and dexamethasone-treated cells, and results showed that expression levels of IL-9, MIP- $1 \beta$, and RANTES were significantly lower in the shikonin group than in the dexamethasone group (Table 6). These data indicate that shikonin treatment can inhibit Der p 2-induced proinflammatory cytokine expression and exhibit better results than steroids in some patients with $\mathrm{AD}$.

\section{Discussion}

Numerous experiments and clinical trials are in progress for developing new drugs to treat AD. However, only dupilumab, a monoclonal antibody against IL-4RA, has been approved by the United States FDA in recent years. The high cost and high risk of life-threatening infection cause concern and discontent among patients with AD. Partial AD patients still showed poor response to biologics. Thus, to discover a new inexpensive drug with fewer side effects becomes necessary. In this study, our data revealed that shikonin reduced Der $\mathrm{p}$ 2-induced Th2-, Th17-, and macrophagerelated cytokine expression in dendritic cells isolated from HDM-allergic patients with AD.

IL-1 receptors mediated multiple proinflammatory responses, including enhancing expression of cytokines related to Th2 and Th17 as well as inducing proinflammatory cytokine production [21-23]. IL-1 receptors can conjugate with different cytokines such as IL-1 $\beta$, IL-18, and IL-33 [24]. After activation by the agonist, the signaling pathway initiated transcription of proinflammatory cytokines, leading to inflammatory responses [25]. In normal homeostasis, when cells receive excessive inflammatory stimuli, they start creating negative feedback loops to offset inflammation. In the case of a skin disorder, activated keratinocytes produce a neuropeptide to enhance IL-10 expression for creating negative feedback signals for modulating inflammatory responses in the skin [26]. In the present study, we found that the basal level of IL-1ra in patients with AD was significantly higher than that in the controls. IL-1ra has been reported to bind to IL-1RI and block the activity of IL- $1 \alpha$ or IL-1 $\beta$ [27]. This result suggested that IL-1ra served as a negative regulator, and its increased expression in patients with $\mathrm{AD}$ was expected to inhibit inflammatory responses.

Proinflammatory cytokines secreted by Th2 cells, Th17 cells, and macrophages play a major role in $\mathrm{AD}$ pathogenesis. The levels of IL-4, IL-5, and IL-13 have been reported to be higher in the skin of patients with $A D$ and upregulated $\operatorname{IgE}$ levels were correlated with higher 


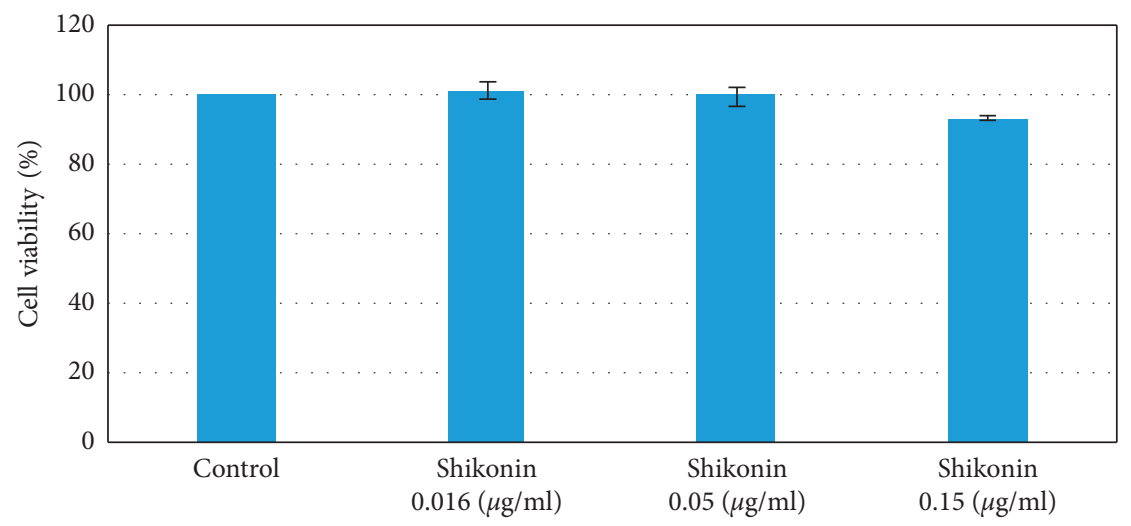

FIgure 2: Cytotoxic effect of shikonin on dendritic cells isolated from patients with AD. Dendritic cells were treated with $0.016,0.05$, and $0.15 \mu \mathrm{g} / \mathrm{mL}$ of shikonin for 24 hours. After treatment, cell viability was assessed using the trypan blue exclusion assay.

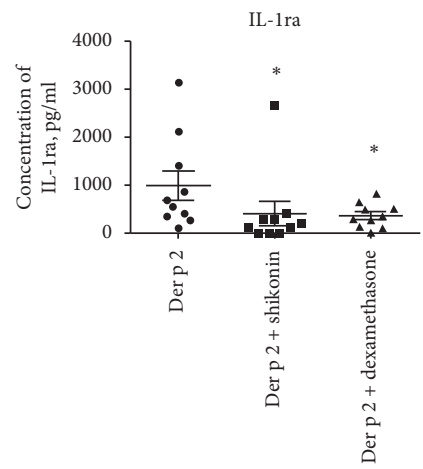

(a)

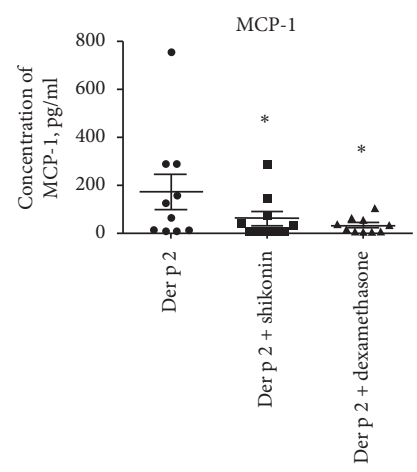

(e)

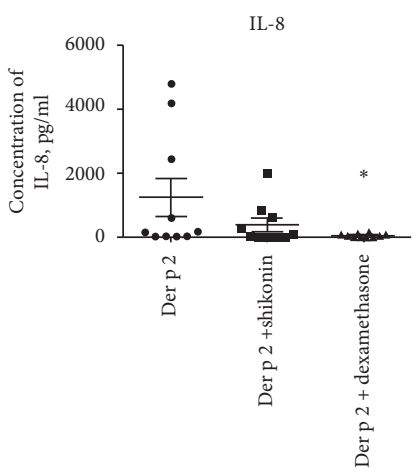

(b)

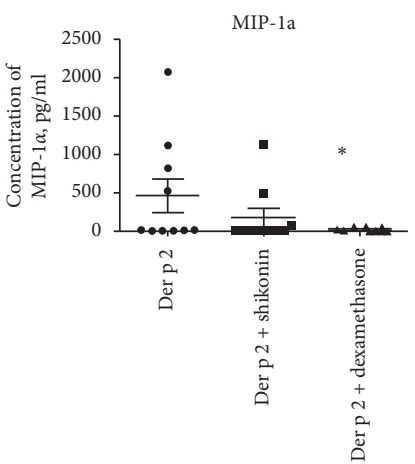

(f)

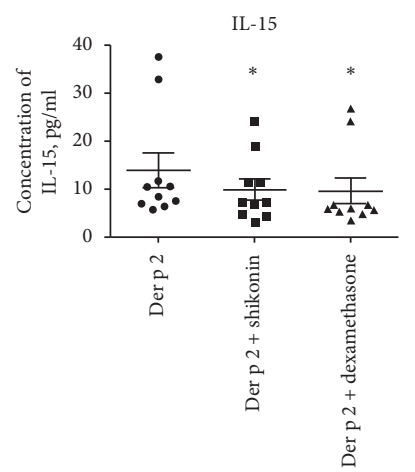

(c)

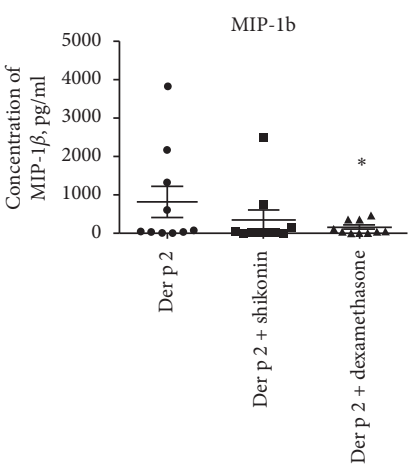

(g)

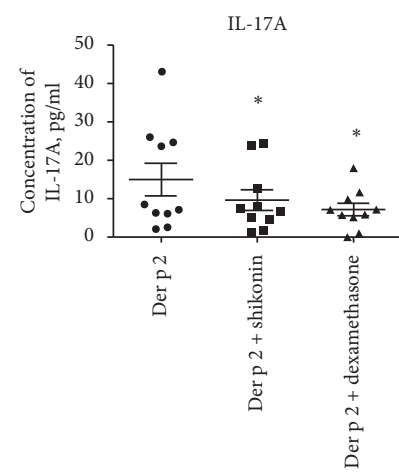

(d)

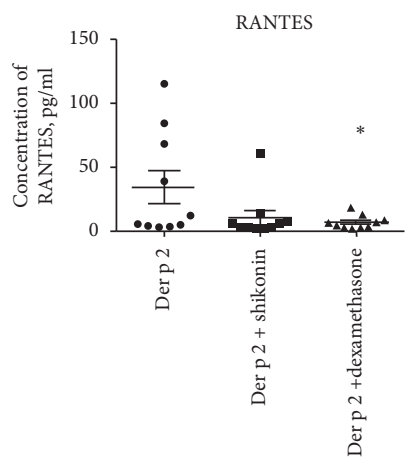

(h)

FIGURE 3: Effects of shikonin on inhibiting proinflammatory cytokine and chemokine expression. Dendritic cells isolated from patients with $\mathrm{AD}$ and incubated with $1.5 \mathrm{~g} / \mathrm{mL}$ of Der p 2; $1.5 \mathrm{~g} / \mathrm{mL}$ of Der p 2 and $0.05 \mathrm{~g} / \mathrm{mL}$ of shikonin; and $1.5 \mathrm{~g} / \mathrm{mL}$ of Der $\mathrm{p} 2$ and $10-7 \mathrm{M}$ dexamethasone for 24 hours. Culture supernatants were collected, and cytokine and chemokine expression were measured using the multiplex assay system. (a) IL-1ra, (b) IL-8, (c) IL-15, (d) IL-17A, (e) MCP-1, (f) MIP-1a, (g) MIP-1b, (h) RANTES.

expression of IL-5 and IL-13 in patients with AD [28]. Overexpression of Th2 cell-related cytokines was reported to cause epidermal thickening, inflammation, eosinophilia, pruritus and magnify the symptoms of AD [29]. By contrast, Th17 overactivation was observed in Asian patients with AD and early-onset pediatric patients with AD [30]. Th17 cells express proinflammatory cytokines, such as IL-17A and IL22 , and their differentiation is regulated by IL-6, TGF- $\beta$, and IL-1 $\beta$ [31]. IL-17 can stimulate epithelial cells and fibroblasts to produce cytokines and chemokines, such as IL-6 and IL-8, attract additional immune cells, infiltrate into skin lesions, and cause cutaneous remodeling in AD. Overexpression of IL-17 has been reported to enhance fibrosis and chronic dermatitis and stimulate eosinophils to secrete other chemokines, which causes exacerbation of dermatitis [32, 33]. In our data, shikonin and dexamethasone reduced expression of Th17-related cytokines, IL- $1 \beta$, IL- 6 , and IL-17 and that of Th2-related cytokines, IL- 6 and IL-9, which were secreted by dendritic cells. By contrast, minimal levels of IL4, IL-5, and IL13 were observed, which was consistent with 
TABle 4: Proinflammatory cytokine levels of the Der p 2 group and shikonin-treated group $(n=6)$.

\begin{tabular}{lccccc}
\hline & Der p 2 & & Shikonin & \\
& Mean & SD & Mean & SD & \pm 0.4 \\
IL-4 & 0.5 & \pm 0.4 & 0.3 & \pm 1.1 & 0.138 \\
IL-5 & 0.7 & \pm 1.7 & 0.9 & \pm 0.7 & 0.715 \\
IL-6 & 3.3 & \pm 4.1 & 0.7 & \pm 5.3 & $0.043^{*}$ \\
IL-9 & 21.6 & \pm 22.6 & 5.3 & \pm 1.5 & $0.028^{*}$ \\
IL-13 & 1.3 & \pm 2.2 & 1.2 & \pm 2.6 & 0.893 \\
IL-17A & 12.1 & \pm 9.6 & 5.5 & \pm 14.5 & $0.027^{*}$ \\
MCP-1 & 91.0 & \pm 112.9 & 15.6 & \pm 6.7 & $0.028^{*}$ \\
MIP-1 $\alpha$ & 330.6 & \pm 505.5 & 9.9 & \pm 7.2 & $0.046^{*}$ \\
MIP-1 $\beta$ & 610.2 & \pm 919.4 & 6.9 & $0.028^{*}$ \\
RANTES & 29.8 & \pm 36.4 & 3.6 & \pm 2.0 & $0.028^{*}$ \\
\hline
\end{tabular}

Data are expressed as the mean \pm standard deviation (SD). Data were analyzed using the Wilcoxon signed-rank test.

Table 5: Proinflammatory cytokine level of the Der p 2 group and dexamethasone-treated group $(n=6)$.

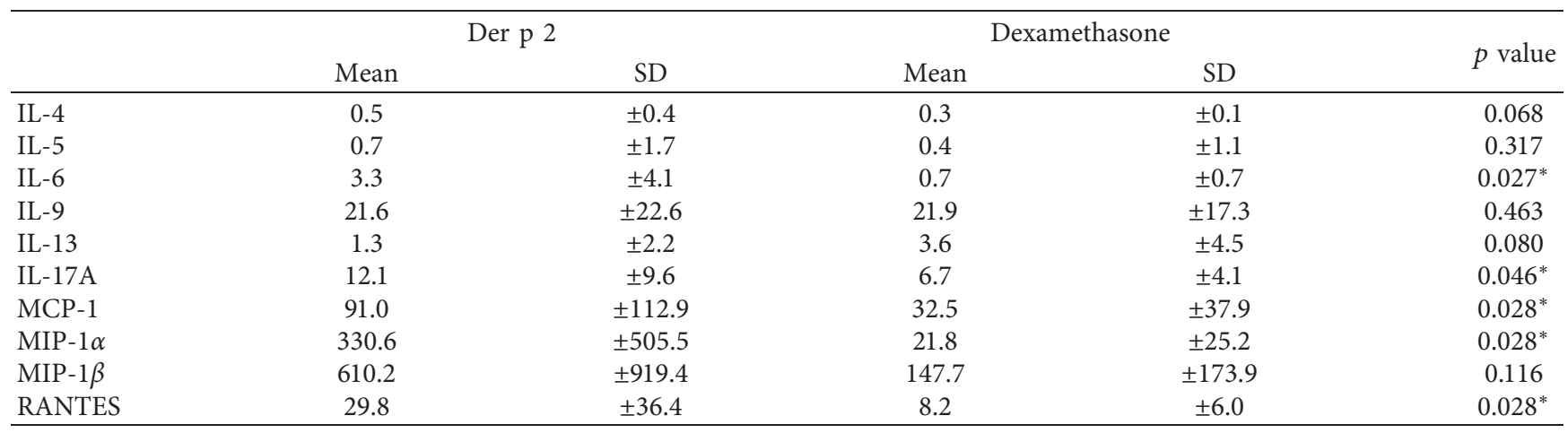

Data are expressed as the mean \pm standard deviation (SD). Data were analyzed using the Wilcoxon signed-rank test.

TABle 6: Proinflammatory cytokine levels of the shikonin-treated group and dexamethasone-treated group $(n=6)$.

\begin{tabular}{|c|c|c|c|c|c|}
\hline & \multicolumn{2}{|c|}{ Shikonin } & \multicolumn{2}{|c|}{ Dexamethasone } & \multirow{2}{*}{$p$ value } \\
\hline & Mean & $\mathrm{SD}$ & Mean & SD & \\
\hline IL-4 & 0.33 & \pm 0.36 & 0.26 & \pm 0.14 & 0.465 \\
\hline IL-5 & 0.90 & \pm 1.09 & 0.44 & \pm 1.08 & 0.068 \\
\hline IL-6 & 0.74 & \pm 0.73 & 0.75 & \pm 0.66 & 1.000 \\
\hline IL-9 & 5.26 & \pm 5.31 & 21.90 & \pm 17.35 & $0.046^{*}$ \\
\hline IL-13 & 1.23 & \pm 1.46 & 3.63 & \pm 4.52 & 0.075 \\
\hline IL-17A & 5.53 & \pm 2.56 & 6.73 & \pm 4.12 & 0.249 \\
\hline MCP-1 & 15.58 & \pm 14.45 & 32.52 & \pm 37.89 & 0.116 \\
\hline MIP- $1 \alpha$ & 9.91 & \pm 6.72 & 21.77 & \pm 25.23 & 0.463 \\
\hline MIP- $1 \beta$ & 6.92 & \pm 7.17 & 147.70 & \pm 173.91 & $0.046^{*}$ \\
\hline RANTES & 3.60 & \pm 2.03 & 8.23 & \pm 5.97 & $0.043^{*}$ \\
\hline
\end{tabular}

Data are expressed as the mean \pm standard deviation (SD). Data were analyzed using the Wilcoxon signed-rank test.

previous reports in which no secretion by dendritic cells was observed [34]. Shikonin and dexamethasone attenuated IL-6 and IL-9 secretion by dendritic cells, which can modulate the Th2 pathway and control AD. Shikonin downregulated IL-9 more effectively than dexamethasone in 6 of the 10 patients. Tracing back our clinical data, the 10 patients responded satisfactorily to steroids; this result was comparable to those of our dendritic cytokine and chemokine tests. Two patients (patient 7 and 8) were treated using TYO; they exhibited a highly positive response to clinical treatment. Both of them responded more satisfactorily to TYO than to topical steroids subjectively and objectively. Patient 3 also used TYO, but his condition deteriorated. The clinical response of Patient 3 to TYO was consistent with the upregulating effects of shikonin. Dendritic cells activated by Der p 2-related cytokines, such as MCP-1, MIP- $1 \alpha$, and MIP- $1 \beta$, can stimulate macrophages to secrete increased amounts of cytokines and chemokines to attract additional immune cells to infiltrate skin lesions and form a positive loop, leading to severe dermatitis $[35,36]$. Furthermore, the effects of IL-15 


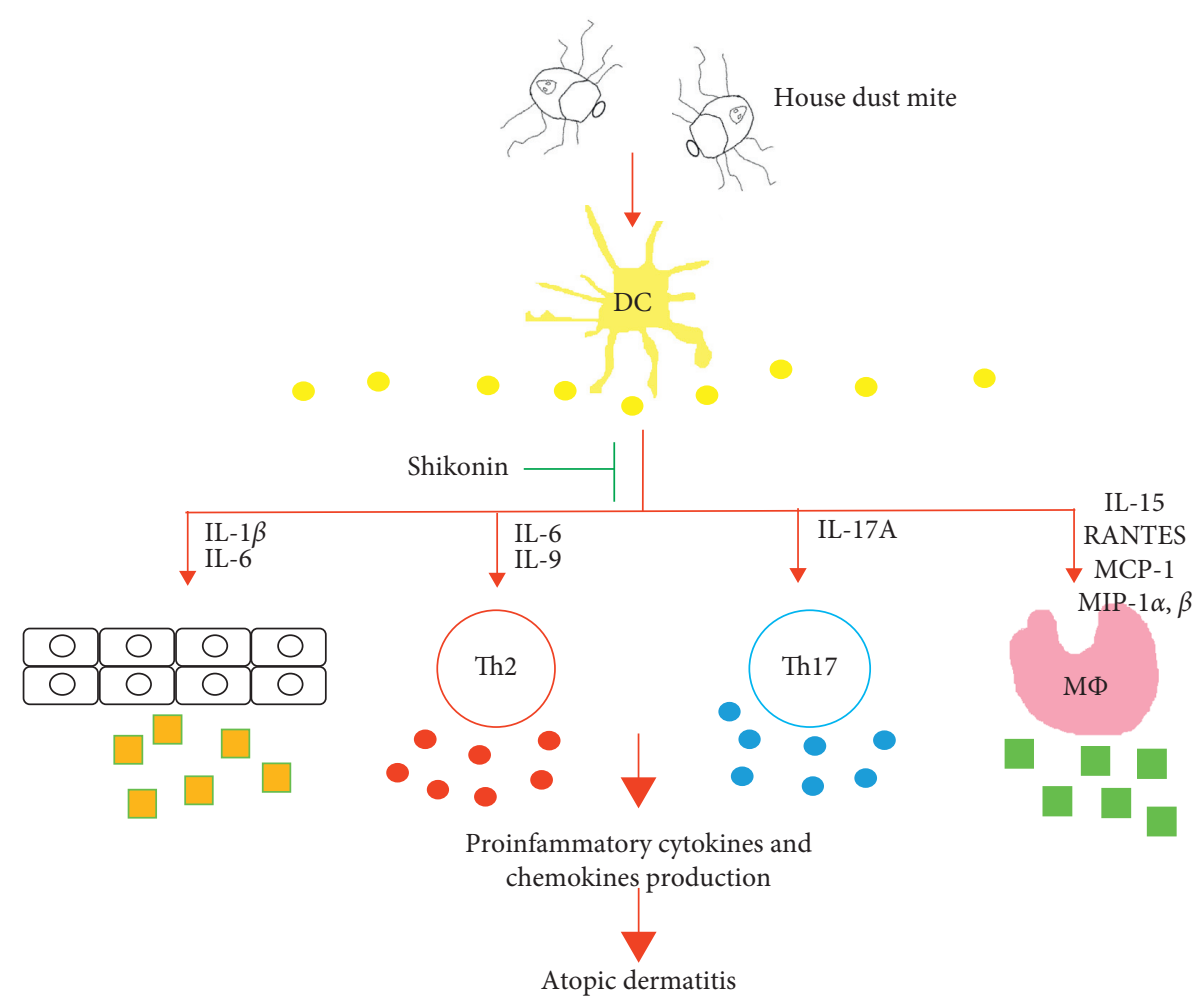

Figure 4: Schematic diagram of the proposed action of shikonin om Der p 2-induced dendritic cell activation and proinflammatory cytokine production.

secretion by activated macrophages and dendritic cells remain controversial. Decreased IL-15 expression has been reported to contribute to the pathogenesis of $\mathrm{AD}$ by enhancing IgE production [37]. By contrast, IL-15 could enhance T-cell recruitment and survival in the skin [38]. Our results showed that shikonin treatment could significantly reduce Der p 2-induced MCP-1, MIP- $1 \alpha$, MIP- $1 \beta$, and IL-15 expression in dendritic cells isolated from HDM-allergic patients with $\mathrm{AD}$. It suggested that shikonin could attenuate chemokine orchestration. RANTES is a potent chemoattractant for eosinophils, monocytes, basophils, and lymphocytes. Serum RANTES levels are correlated with IgE levels, total eosinophil counts, and lactate dehydrogenase levels [39]. It plays a major role in eosinophil infiltration and augmentation in disease severity [40]. In our study, RANTES was secreted by Der p 2-activated dendritic cells and downregulated by shikonin. Dendritic cells doubtlessly play an important role in connecting the innate and adaptive immune systems [41]. In this study, dendritic cells were the focus for investigating the efficacy of shikonin on inhibiting Der p 2-induced proinflammatory cytokine and chemokine expression. In the present study, we demonstrated that shikonin reduced the expression of the proinflammatory cytokines IL-6 and IL-17A and production of the macrophage-related cytokines and chemokines MCP1 , MIP- $1 \alpha$, and MIP-1 $\beta$. Moreover, shikonin inhibited production of the proinflammatory cytokines IL-9, MIP-1 $\beta$, and RANTES more effectively than dexamethasone. In the recent year, CCL17 and CCL22 have been reported that played an important role in Th2 cytokines induced AD [42]. CCL17 and CCL22 could be secreted by dendritic cells not only developed the inflammatory responses but also became pivotal regulator of the AD pathogenesis. These chemokines expression regulated by shikonin in our model will be investigated in the future. The schematic diagram of how shikonin ameliorates dendritic cell activation and treats $\mathrm{AD}$ is shown in Figure 4.

According to in vitro and clinical data, shikonin can regulate the overactivated immune response in patients with $\mathrm{AD}$ and act as an alternative medication to treat $\mathrm{AD}$ with lower risks and fewer side effects than the current medications.

The effects of shikonin on inhibiting Der p 2-induced proinflammatory cytokine expression and dermatitis in patients with $\mathrm{AD}$ have been demonstrated in this study. However, this study has some limitations. First, dendritic cells were isolated from only 10 patients with $\mathrm{AD}$; however, the expression of proinflammatory cytokines could not be inhibited in three patients and only one patient exhibited trace inhibition effects after cells were cocultured with Der $\mathrm{p}$ 2 and shikonin (data not shown). Interindividual variability in response to the same drug could be due to physiological variables, pathological factors, genetic variation of key enzymes of receptors, and interaction with other drugs [43]. This variability also revealed the importance of precision medical treatment for reducing $\mathrm{AD}$, and the methodology we used in this study could be used as a platform for screening patients with $\mathrm{AD}$ with a satisfactory response to the shikonin treatment. Second, in this study, only 10 
patients with $\mathrm{AD}$ were enrolled to evaluate the effects of shikonin on inhibiting proinflammatory cytokine production. Dendritic cell samples isolated from a large number of patients with $\mathrm{AD}$ and more dendritic cells expressed chemokines such as CCL17 and CCL22 which were related to $\mathrm{AD}$ pathogenesis should be investigated in future studies. Furthermore, clinical trials should be used to evaluate the direct effects of shikonin on ameliorating dermatitis of patients with $\mathrm{AD}$. Third, the pathogenesis of $\mathrm{AD}$ is highly complicated, and different immune cells, skin microorganisms, and skin barriers are involved. Therefore, the effects of shikonin on other immune cells and keratinocytes as well as modulating the composition of microorganisms should be validated in future studies.

\section{Conclusion}

In conclusion, to our knowledge, this is the first report on shikonin-mediated inhibition of expression of proinflammatory cytokines by dendritic cells in patients with $\mathrm{AD}$. Through this study, we provided a novel screening platform for individual treatment and evidence that shikonin is a promising potential therapy for $\mathrm{AD}$.

\section{Data Availability}

The data used to support the findings of this study are available from the corresponding author upon request.

\section{Conflicts of Interest}

The authors declare no conflicts of interest.

\section{Authors' Contributions}

C-Y Yen and S-J Yu collected data from patients with atopic dermatitis and wrote the manuscript, W-D Chiang and S-J Yu participated in experimentation, S-Y Liu performed cell experiment and the measurement of cytokines and chemokine, and C-L Hsieh participated in the protocol design and revised the manuscript.

\section{Acknowledgments}

The authors sincerely thank the Biostatistics Task Force and the Center for Translational Medicine of Taichung Veterans General Hospital, Taichung, Taiwan, Republic of China. This work was financially supported by the grant from Taichung Veterans General Hospital, Taiwan (TCVGH-T1097804) and Chinese Medicine Research Center, China Medical University from the Featured Areas Research Center Program within the framework of the Higher Education Sprout Project by the Ministry of Education (MOE) in Taiwan (CMRC-CENTER-0).

\section{References}

[1] S. Barbarot, S. Auziere, A. Gadkari et al., "Epidemiology of atopic dermatitis in adults: results from an international survey," Allergy, vol. 73, no. 6, pp. 1284-1293, 2018.
[2] J. Harrop, S. Chinn, G. Verlato et al., "Eczema, atopy and allergen exposure in adults: a population-based study," Clinical \& Experimental Allergy, vol. 37, no. 4, pp. 526-535, 2007.

[3] K. Bingefors, Å. Svensson, D. Isacson, and M. Lindberg, "Selfreported lifetime prevalence of atopic dermatitis and comorbidity with asthma and eczema in adulthood: a population-based cross-sectional survey," Acta Dermato Venereologica, vol. 93, no. 4, pp. 438-441, 2013.

[4] T. Muto, S. D. Hsieh, Y. Sakurai et al., "Prevalence of atopic dermatitis in Japanese adults," British Journal of Dermatology, vol. 148, no. 1, pp. 117-121, 2003.

[5] M. S. Ng, S. Tan, N. H. Chan, A. Y. Foong, and M. J. Koh, "Effect of atopic dermatitis on quality of life and its psychosocial impact in Asian adolescents," Australasian Journal of Dermatology, vol. 59, no. 2, pp. e114-e117, 2018.

[6] Y. M. F. Andersen, A. Egeberg, L. Skov, and J. P. Thyssen, "Comorbidities of atopic dermatitis: beyond rhinitis and asthma," Current Dermatology Reports, vol. 6, no. 1, pp. 35-41, 2017.

[7] A. S. Adamson, "The economics burden of atopic dermatitis," Advances in Experimental Medicine and Biology, vol. 1027, pp. 79-92, 2017.

[8] T. Hajar, J. R. V. Gontijo, and J. M. Hanifin, "New and developing therapies for atopic dermatitis," Anais Brasileiros De Dermatologia, vol. 93, no. 1, pp. 104-107, 2018.

[9] D. D’Ippolito and M. Pisano, "Dupilumab (dupixent): an interleukin-4 receptor antagonist for atopic dermatitis," $P$ \& T: a Peer-Reviewed Journal for Formulary Management, vol. 43, no. 9, pp. 532-535, 2018.

[10] S. R. Feldman, L. S. Cox, L. C. Strowd et al., "The challenge of managing atopic dermatitis in the United States," American Health \& Drug Benefits, vol. 12, no. 2, pp. 83-93, 2019.

[11] N. Novak, "An update on the role of human dendritic cells in patients with atopic dermatitis," Journal of Allergy and Clinical Immunology, vol. 129, no. 4, pp. 879-886, 2012.

[12] W. Peng and N. Novak, "Pathogenesis of atopic dermatitis," Clinical \& Experimental Allergy, vol. 45, no. 3, pp. 566-574, 2015.

[13] S. Kasraie and T. Werfel, "Role of macrophages in the pathogenesis of atopic dermatitis," Mediators of Inflammation, vol. 2013, Article ID 942375, 15 pages, 2013.

[14] C.-Y. Yen and C.-L. Hsieh, "Therapeutic effect of Tzu-Yun ointment on patients with atopic dermatitis: a preliminary, randomized, controlled, open-label study," The Journal of Alternative and Complementary Medicine, vol. 22, no. 3, pp. 237-243, 2016.

[15] C.-C. Lee, C.-N. Wang, Y.-T. Lai et al., "Shikonin inhibits maturation of bone marrow-derived dendritic cells and suppresses allergic airway inflammation in a murine model of asthma," British Journal of Pharmacology, vol. 161, no. 7, pp. 1496-1511, 2010.

[16] L. S. Yoshida, T. Kakegawa, Y. Yuda, and H. Takano-Ohmuro, "Shikonin changes the lipopolysaccharide-induced expression of inflammation-related genes in macrophages," Journal of Natural Medicines, vol. 71, no. 4, pp. 723-734, 2017.

[17] D.-P. Yuan, L. Gu, J. Long et al., "Shikonin reduces endometriosis by inhibiting RANTES secretion and mononuclear macrophage chemotaxis," Experimental and Therapeutic Medicine, vol. 7, no. 3, pp. 685-690, 2014.

[18] H. Guo, J. Sun, D. Li et al., "Shikonin attenuates acetaminophen-induced acute liver injury via inhibition of oxidative stress and inflammation," Biomedicine \& Pharmacotherapy, vol. 112, Article ID 108704, 2019. 
[19] J. M. R. Hanifin and G. Rajka, "Diagnostic features of atopic dermatitis," Acta dermato-venereologica. Supplementum, vol. 92, pp. 44-47, 1980.

[20] S. J. Yu, E. C. Liao, M. L. Sheu, D. T. Chang, and J. J. Tsai, "Correction: correction: cell-penetrating peptide derived from human eosinophil cationic protein inhibits mite allergen der $p$ 2 induced inflammasome activation," PLoS One, vol. 10, no. 6, Article ID e0129187, 2015.

[21] S. M. Caucheteux, J. Hu-Li, L. Guo et al., "IL-1 $\beta$ enhances inflammatory Th2 differentiation," Journal of Allergy and Clinical Immunology, vol. 138, no. 3, pp. 898-901 e4, 2016.

[22] Y. Sha and S. Markovic-Plese, "A role of IL-1R1 signaling in the differentiation of Th17 cells and the development of autoimmune diseases," Self/Nonself, vol. 2, no. 1, pp. 35-42, 2011.

[23] L. E. Jensen, "Targeting the IL-1 family members in skin inflammation," Current Opinion in Investigational Drugs (London, England: 2000), vol. 11, no. 11, pp. 1211-1220, 2010.

[24] C. Gabay, C. Lamacchia, and G. Palmer, "IL-1 pathways in inflammation and human diseases," Nature Reviews Rheumatology, vol. 6, no. 4, pp. 232-241, 2010.

[25] M. Bernard, C. Carrasco, L. Laoubi et al., "IL-1 $\beta$ induces thymic stromal lymphopoietin and an atopic dermatitis-like phenotype in reconstructed healthy human epidermis," The Journal of Pathology, vol. 242, no. 2, pp. 234-245, 2017.

[26] K. Seiffert and R. D. Granstein, "Neuropeptides and neuroendocrine hormones in ultraviolet radiation-induced immunosuppression," Methods, vol. 28, no. 1, pp. 97-103, 2002.

[27] J. H. Lee, L. C. Wang, H. H. Yu, Y. T. Lin, Y. H. Yang, and B. L. Chiang, "Type I IL-1 receptor (IL-1RI) as potential new therapeutic target for bronchial asthma," Mediators of Inflammation, vol. 2010, Article ID 567351, 7 pages, 2010.

[28] C.-W. Jeong, K.-S. Ahn, N.-K. Rho et al., "Differential in vivo cytokine mRNA expression in lesional skin of intrinsic vs. extrinsic atopic dermatitis patients using semiquantitative RT-PCR," Clinical Experimental Allergy, vol. 33, no. 12, pp. 1717-1724, 2003.

[29] E. B. Brandt and U. Sivaprasad, "Th2 cytokines and atopic dermatitis," Journal of Clinical \& Cellular Immunology, vol. 2, no. 3, p. 110, 2011

[30] S. Noda, M. Suarez-Farinas, B. Ungar et al., "The Asian atopic dermatitis phenotype combines features of atopic dermatitis and psoriasis with increased Th17 polarization," Journal of Allergy and Clinical Immunology, vol. 136, no. 5, pp. 12541264, 2015.

[31] C. T. Weaver, R. D. Hatton, P. R. Mangan, and L. E. Harrington, "IL-17 family cytokines and the expanding diversity of effector T cell lineages," Annual Review of Immunology, vol. 25, no. 1, pp. 821-852, 2007.

[32] P. F. Y. Cheung, C. K. Wong, and C. W. K. Lam, "Molecular mechanisms of cytokine and chemokine release from eosinophils activated by IL-17A, IL-17F, and IL-23: implication for Th17 lymphocytes-mediated allergic inflammation," The Journal of Immunology, vol. 180, no. 8, pp. 5625-5635, 2008.

[33] S. Molet, Q. Hamid, F. Davoine et al., "IL-17 is increased in asthmatic airways and induces human bronchial fibroblasts to produce cytokines," Journal of Allergy and Clinical Immunology, vol. 108, no. 3, pp. 430-438, 2001.

[34] B. Nedoszytko, M. Sokolowska-Wojdylo, K. RuckemannDziurdzinska, J. Roszkiewicz, and R. J. Nowicki, "Chemokines and cytokines network in the pathogenesis of the inflammatory skin diseases: atopic dermatitis, psoriasis and skin mastocytosis," Advances in Dermatology and Allergology, vol. 31, no. 2, pp. 84-91, 2014.
[35] M. L. Giustizieri, F. Mascia, A. Frezzolini et al., "Keratinocytes from patients with atopic dermatitis and psoriasis show a distinct chemokine production profile in response to T cellderived cytokines," Journal of Allergy and Clinical Immunology, vol. 107, no. 5, pp. 871-877, 2001.

[36] B. Homey, M. Steinhoff, T. Ruzicka, and D. Y. Leung, "Cytokines and chemokines orchestrate atopic skin inflammation," Journal of Allergy and Clinical Immunology, vol. 118, no. 1, pp. 178-189, 2006.

[37] P. Y. Ong, Q. A. Hamid, J. B. Travers et al., "Decreased IL-15 may contribute to elevated IgE and acute inflammation in atopic dermatitis," The Journal of Immunology, vol. 168, no. 1, pp. 505-510, 2002.

[38] C. C. Chu, P. Di Meglio, and F. O. Nestle, "Harnessing dendritic cells in inflammatory skin diseases," Seminars in Immunology, vol. 23, no. 1, pp. 28-41, 2011.

[39] Y. Kaburagi, Y. Shimada, T. Nagaoka, M. Hasegawa, K. Takehara, and S. Sato, "Enhanced production of CCchemokines (RANTES, MCP-1, MIP-1 alpha, MIP-1 beta, and eotaxin) in patients with atopic dermatitis," Archives of Dermatological Research, vol. 293, no. 7, pp. 350-355, 2001.

[40] Y. Kato, R. Pawankar, Y. Kimura, and S. Kawana, "Increased expression of RANTES, CCR3 and CCR5 in the lesional skin of patients with atopic eczema," International Archives of Allergy and Immunology, vol. 139, no. 3, pp. 245-257, 2006.

[41] T. Bieber, N. Novak, N. Herrmann, and S. Koch, "Role of dendritic cells in atopic dermatitis: an update," Clinical Reviews in Allergy \& Immunology, vol. 41, no. 3, pp. 254-258, 2011.

[42] H. S. Lim, C. S. Seo, S. E. Jin et al., "Ma Huang tang suppresses the production and expression of inflammatory chemokines via downregulating STAT1 phosphorylation in HaCaT keratinocytes," Evidence Based Complementary and Alternative Medicine, vol. 2016, Article ID 7831291, 8 pages, 2016.

[43] B. Rocca and C. Patrono, "Determinants of the interindividual variability in response to antiplatelet drugs," Journal of Thrombosis and Haemostasis, vol. 3, no. 8, pp. 1597-1602, 2005. 